\title{
Preoperative Karnofsky Performance
}

\section{Status}

National Cancer Institute

\section{Source}

National Cancer Institute. Preoperative Karnofsky Performance Status. NCI Thesaurus.

Code C158661.

The Karnofsky Performance Status Score assessed preoperatively. 